\section{Acute lymphoblastic leukaemia in a patient with cardiofaciocutaneous syndrome}

EDITOR-Cardiofaciocutaneous syndrome (CFC syndrome) is a multiple congenital abnormality syndrome associated with characteristic facial appearance, congenital heart defects, ectodermal anomalies, and growth failure. The most frequent cardiac anomalies are pulmonary stenosis and atrial septal defect. The facial characteristics are high forehead, hypoplasia of the supraorbital ridges, downward slanting palpebral fissures, depressed nasal bridge, and posteriorly angulated ears with prominent helices. The often woolly hair is sparse and friable. The skin shows hyperkeratosis that can be as severe as generalised ichthyosis.' There is an assumption that Noonan syndrome (NS) and CFC syndrome are closely linked. However, in NS there is a link with malignancies, but in CFC syndrome no descriptions of cases with a malignancy have been published.

We report a 5 year old girl of unrelated, white parents (at birth paternal age was 47 years and maternal age was 29 years) with CFC syndrome, who presented with acute lymphoblastic leukaemia (ALL).

During the pregnancy there was polyhydramnios. Delivery at 40 weeks was uncomplicated and birth weight was $3500 \mathrm{~g}$. At the age of 10 months the diagnosis of CFC syndrome was made based on dysmorphic features, cardiac anomalies, and skin abnormalities. Cardiac features included an axis in the left upper quadrant, with low voltages over the left precordial leads on ECG, mild pulmonary stenosis (an estimated gradient of $16 \mathrm{~mm} \mathrm{Hg}$ ), and an atrial septal defect on Doppler echocardiography. On repeated investigation at the age of $31 / 2$ years the pulmonary stenosis was no longer detectable but the atrial septal defect with left to right shunt was still noted. In addition, asymmetrical hypertrophy of the interventricular septum directed towards the left ventricular outflow tract was noted. Initially, at the age of 10 months, skin anomalies were confined to mild hyperkeratosis. Biopsy showed acrokeratosis verruciformis. At the age of 3 years, disseminated keratotic follicular changes had developed, compatible with keratosis pilaris.

Additional investigations included a skeletal survey, which showed no anomalies and a normal bone age, and a cerebral MRI scan at the age of 1 year, showing delayed and irregular myelinisation, widening of the subarachnoid space, and slightly enlarged ventricles. Ophthalmological investigations were normal.

On examination when ALL was diagnosed, weight was $23.5 \mathrm{~kg}$ (>98th centile), height was $110.5 \mathrm{~cm}$ (50th centile), and head circumference was $53 \mathrm{~cm}$ (90th centile). The following anomalies related to the CFC syndrome were present: a high and prominent forehead, woolly, sparse hair, moderately low set ears with prominent helices, pits in both helices, hypertelorism, absent eyebrows, epicanthic folds, mildly downward slanting palpebral fissures, depressed nasal bridge, broad mouth, shield thorax, mild joint laxity, and four café au lait spots $(2 \mathrm{~cm})$ on the trunk and right leg (fig 1). Her developmental age was estimated to be $1 \frac{1 / 2}{2}$ years. Anomalies related to the acute lymphoblastic leukaemia were hepato- and splenomegaly. Lymphoblasts were seen in the peripheral blood ( $8 \%$ of $1.4 \times 10^{9} / 1$ leucocytes). Bone marrow aspirate showed $98 \%$ lymphoblasts positive for TdT, HLA-Dr, CD 34, CD13, CD33, CD19, CD10, CD22, and CD79
F Med Genet 1999;36:799-800 and negative for cytoplasmic IgM and membrane $\operatorname{IgM},-\kappa$, $-\lambda$, CD 15, CD 65, myeloperoxidase, and $T$ cell markers. Cytogenetic analysis showed 45-46,XX,add(3)(p 14), $\operatorname{del}(9)$

(p21 p22), +10,t(12;21)(p13;22), +del(12)(p11;p12), del (15)(q13q24), der(16;19)(q10;p10), del(22)(q1 1 q13) [27] and 46,XX[13]. FISH studies were negative for BCR/ABL fusion and positive for TELAAML1 fusion. Examination of cerebrospinal fluid showed no lymphoblasts.

Induction therapy consisted of vincristine, dexamethasone, and $\mathbf{E}$ coli asparaginase; remission was achieved in five weeks. The very rapid loss of hair during the first two weeks of treatment was striking. For prophylaxis of a central nervous system relapse methotrexate was given. At present the patient is still on maintenance treatment, which is composed of vincristine, dexamethasone, 6-mercaptopurine, and methotrexate.

In 1996 Krajewska-Walasek et af summarised the anomalies related to CFC. Common symptoms, also present in our patient, are polyhydramnios, psychomotor retardation, relative macrocephaly, cortical brain atrophy, abnormal hair, skin anomalies, cardiac defects, shield thorax, and joint laxity. Pulmonary stenosis, atrial septal defect, and hypertrophic cardiomyopathy are the most frequent cardiac defects reported in CFC. The leukaemia in the present patient was an ALL of common phenotype. Findings, including the TEL/AMLl fusion, which is noted in $20-30 \%$ of childhood ALI, indicate a favourable ultimate outcome. ${ }^{3}$ CFC cases are mostly sporadic, but autosomal dominant inheritance has been suggested, although the described family may also fit the diagnosis of

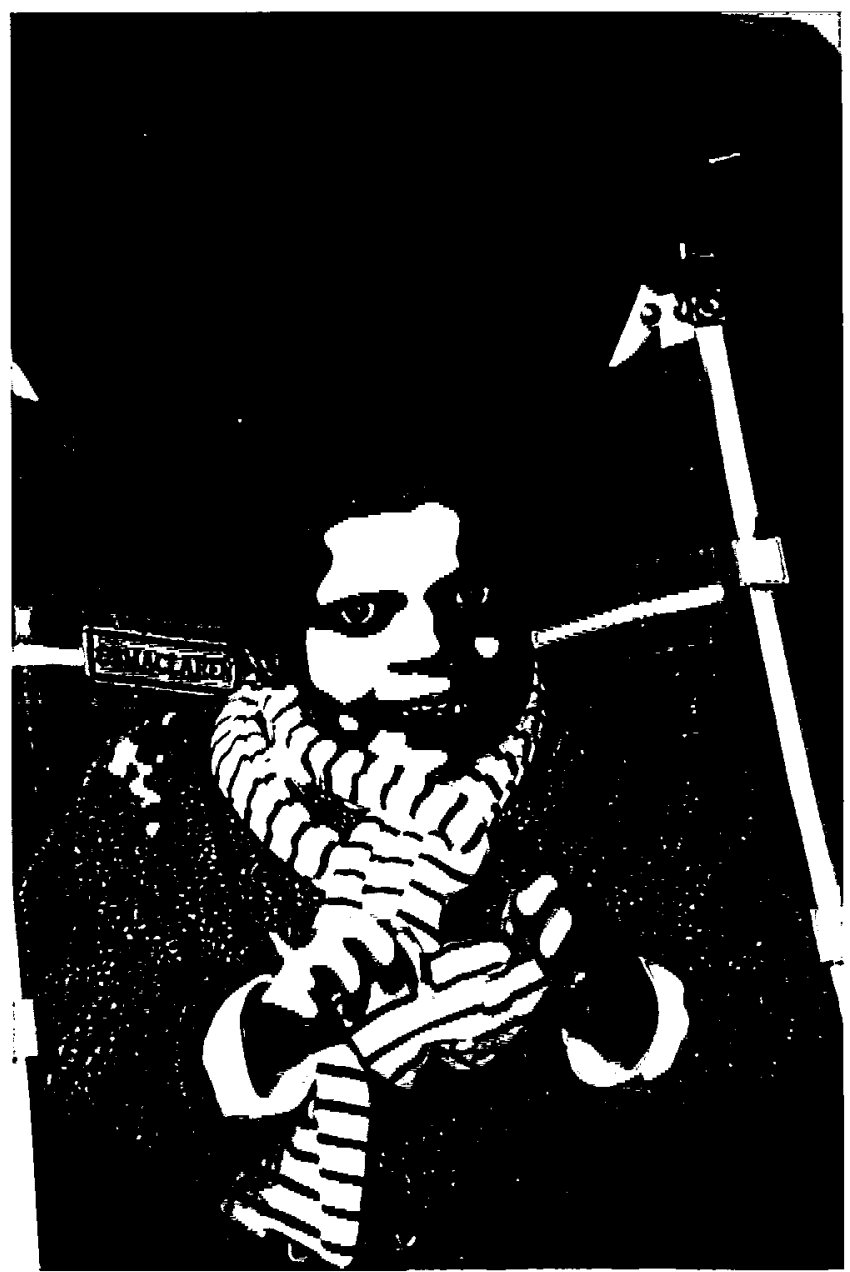

Figure 1 Photograph of proband taken tz:o zeeks before the diagnosis of letkaemia was made. (Pltotograph reproduced with permission.) 
NS. ${ }^{4}$ CFC syndrome has often been compared to NS, which follows an autosomal dominant pattern of inheritance ${ }^{5}$ and half of these patients are sporadic cases. Linkage of NS with a locus on 12q24 has been reported in two families, but in most families no linkage with this locus could be established. ${ }^{6}{ }^{7}$ The similarity of NS to CFC syndrome is especially clear in the facial dysmorphology, the nature of the cardiac defects, and decreased growth. In CFC syndrome additional findings are skin abnormalities, hypotrichosis, mental retardation, and eye anomalies. ${ }^{1}$ These similarities and differences are still a matter of debate; some think that NS and CFC syndrome are genetically different conditions, ${ }^{8}$ and others assume that NS and CFC syndrome are either allelic variants or constitute a contiguous gene syndrome. ${ }^{9-12}$ Recently, in a single family, it was suggested through linkage analysis that NS and $C F C$ resulted from a variable expression of the same genetic defect. ${ }^{7}$

Malignancies have not been previously reported in patients with CFC syndrome, but in NS there are reports of various types of malignancies, including rhabdomyosarcoma, malignant schwannoma, phaeochromocytoma, ganglioneuroma, and myelodysplasia. ALL is reported most frequently. ${ }^{13}{ }^{14}$ An explanation for the discrepancy in the occurrence of malignancies in NS and CFC syndrome might be the small number of patients diagnosed with CFC syndrome, in contrast to the large number of NS patients. Also the low incidence of paediatric malignancies should be considered; in The Netherlands the figure is 13 per 100000 in $0-14$ year olds. ${ }^{15}$ As a result it remains uncertain whether the occurrence of leukaemia in the present case is truly another manifestation of the CFC syndrome or merely coincidence.

We would like to thank C H M Mellink, J H Sillevis Smit, and M A Sobotka-Plojhar for their expert cytogenetic, dermatological, and cardiac analysis.
HENDRIK VAN DEN BERG

Department of Paediatric Oncology, Emma Children Hospital AMIC, Academic Medical Centre, Unizersity of Amsterdam, Meibergdreef 9, 1105 AZ Amsterdam, The Netherlands

RAOUL C M HENNEKAM

Departments of Paediatrics and Human Genetics, Academic Medical Centre, University of Amsterdam, Amsterdam, The Netherlands

1 Reynolds JF, Neri G, Herrmann JP, et al. New multiple congenital anomalies/mental retardation syndrome with cardio-faciocutaneous involvement - the CFC syndrome. Am J Med Genct 1986;25:413-27.

2 Krajewska-Walasek $M$, Chrzanowska $K$, Jastrbska $M$. The cardio-faciocutaneous (CFC) syndrome - two possible new cases and review of the litcrature. Clin Dysmorphol 1996;5:65-72.

3 Shurtleff SA, Buijs A, Behm FG, et al. Tel/AML1 fusion resulting from a cryptic $t(12 ; 21)$ is the most common genetic lesion in pediatric ALL and defines a subgroup of patients with excellent prognosis. Leukemia 1995;9:1985-9.

4 Fryns JP, Volcke P, Van den Berghe H. The cardio-facio-cutaneous (CFC) syndrome: autosomal dominant inheritance in a large family. Genet Couns syndrome: auros

5 Allanson JE. Noonan syndrome 7 Med Genet 1987;24:9-13.

6 Jamieson CR, Van der Burgt I, Brady AF, $c t$ al. Mlapping a gene for Noonan syndrome to the long arm of chromosome 12. Nat Gentet 199.4;8:357-60.

7 Legius E, Schollen E, Matthijs G, Frjns JP. Fine mapping of Noonan cardio-faciocutaneous syndrome in a large family. Eur f Hum Genet 1998; 6:32-7.

8 Neri G, Zollino M. More on the Noonan-CFC controversy. Am $\mathcal{Y} M$ fed Genet 1996;65:100.

9 Verloes A, Le Merrer M, Soyeur D, et al. CFC syndrome: a syndrome distinct from Noonan syndrome. Ann Genet 1988;31:230-4.

10 Leichtman LG. Are cardio-facio-cutaneous syndrome and Noonan syndrome distinct? A case of CFC offspring of a mother with Noonan syndrome. Clin Dysmorphol 1996;5:61-4.

11 Fryer AE, Hol PJ, Hughes HE. The cardio-facio-cutaneous (CFC) syndrome and Noonan syndrome: are they the same? Am 7 Aled Genet syndrome and NoO

12 Lorenzett ME, Fryns JP. Retinitis pigmentosa in a young man with Noonan syndrome: further evidence that Noonan syndrome (NS) and the cardio-facio-cutaneous syndrome (CFC) are variable manifestations of the same entity. $A m \mathcal{F}$ Med Genet 1996;65:97-9.

13 Piombo M, Rosanda C, Pasino M, MIarasini M1, Cerruti P, Comelli A. Acute lymphoblastic leukemia in Noonan syndrome: report of two cases. $\Lambda$ fed Pediatr Oncol 1993;21:454-5.

14 Attard-Montalto SP, Kingston JE, Eden T. Noonan's syndrome and acute Iymphoblastic leukemia. Med Pediatr Oncol 1994;23:391-2.

15 Coebergh NWN, Egeler RM. Epidemiologie van kindertumoren. In: Voûte PA, De Kraker J, Caron HN, eds. Kinderoncologie. Houten: Bohn, Stafleu, Van Loghum, 1997:33-49. 